\title{
A Robust Object Tracking Method Using Structural Similarity in Daubechies Complex Wavelet Domain
}

\author{
Anand Singh Jalal and Uma Shanker Tiwary \\ Indian Institute of Information Technology, Allahabad, India \\ \{asjalal, ust\}@iita.ac.in
}

\begin{abstract}
Many of the existing algorithms for object tracking that are based on spatial domain features, fail in the presence of illumination variation or change in appearance or pose or in the presence of noise. To overcome these problems, in this paper, we have proposed a new method of object tracking using structural similarity index in complex wavelet transform domain, which is approximately shift-invariant. The reference object in the initial frame is modeled by a feature vector in terms of the coefficients of Daubechies complex wavelet transform. A similarity measure based on structural similarity index is used to find the object in the current frame. The advantage of using structural similarity index in complex wavelet domain is that it allows small spatial translations, rotations and scaling changes, which are depicted in fig. 1. Experimental results illustrate that the proposed algorithm has good performance in noisy video with significant variations in object's pose and illumination. The search for the candidate subframe is made fast by using the motion prediction algorithm.
\end{abstract}

\section{Introduction}

Tracking an object in a complex environment is a challenging task. The problem becomes hard when applied to real life situations, such as, sport video analysis to extract highlights, surveillance system to know the suspicious activity, traffic monitoring, and human computer interface to assist visually challenged people [1]. Most of the existing algorithms are unable to track objects in the presence of variations in illumination, appearance and camera angle, as most of these algorithms working in spatial domain use features which are sensitive to these variations. The mean shift algorithm widely used for object tracking proposed by [2], is based on color features. They use Bhattacharyya coefficient as a measure of comparability and optimize the search using mean shift technique. One of the problems of using color or a similar quantity as feature is that it is sensitive to the illumination or appearance changes [3]. In recent years the wavelet feature based techniques have gained popularity in object tracking. One of the features of discrete wavelet transform (DWT) is that the spatial information is retained even after decomposition of an image into four different frequency coefficients. However, one of the major problems with real wavelet transform is that it suffers from shift-sensitivity [4]. In [3] an undecimated wavelet packet transform (UWPT) has been used to overcome the problem of shift sensitivity. However, the UWPT expansion is redundant and computationally intensive. A Daubechies complex wavelet transform can be a better solution which is also approximately 
shift-invariant. Not many researchers have explored the application of complex wavelet transform (CxWT) for tracking problems. Recently [5] has shown the applicability of CxWT to denoising and deblurring.

In this paper, we present a new object tracking algorithm using Daubechies complex wavelet coefficients as features to represent the object. We have used a similarity measure based on structural similarity metrics in the complex wavelet domain. The advantage of using structural similarity metrics in complex wavelet domain is to allow imperceptible spatial translations, and also small rotations and scaling changes. By applying all computations in the complex wavelet domain, we can attain high immunity to noise and can work at higher levels to reduce the computations as per requirement of the application.

The remaining part of the paper is organised as follows. Section 2 gives an overview of Daubechies complex wavelet transform. Section 3 presents the structural similarity metrics. Section 4 describes the proposed algorithm. Section 5 contains results over real world video sequences, and finally, Section 6 concludes and discusses the open issues for future research.

\section{Overview of Daubechies Complex Wavelet Transform}

In object tracking we require a feature which remains invariant by translation and rotation because the different video frames may contain a translated and rotated version of the moving object. As Daubechies CxWT is approximately shift-invariant, it can be a good candidate for object tracking.

Any function $f(t)$ can be decomposed into complex scaling function $\phi(t)$ and a mother wavelet $\psi(t)$ as:

$$
f(t)=\sum_{k} c_{k}^{j_{0}} \phi_{j_{0}, k}(t)+\sum_{j=j_{0}}^{j_{\max }-1} d_{k}^{j} \psi_{j, k}(t)
$$

where, $\mathrm{j}_{0}$ is a low resolution level, $\left\{C_{k}^{j_{0}}\right\}$ and $\left\{d_{k}^{j}\right\}$ are known as approximation $\left[\phi(t)=2 \sum_{n} a_{n} \phi(2 t-n)\right]$ and detail coefficients $\left[\psi(t)=2 \sum_{n}(-1)^{n} \overline{a_{1-n}} \phi(2 t-n)\right]$.

Where $\psi(t)$ and $\phi(t)$ shares the same compact support [-N, N+1] and $a_{n} \mathrm{~s}$ are coefficients. The $a_{n}$ s can be real as well as complex valued and $\sum a_{n}=1$.

The Daubechies wavelet bases $\left\{\psi_{j, k}(t)\right\}$ in one dimension are defined through the above scaling function and multiresolution analysis of $L^{2}(\mathfrak{R})$. During the formation of solution if we relax the Daubechies condition for $a_{n}$ [5], it leads to complex valued scaling function. We have used this symmetric Daubechies complex wavelet transform for tracking.

\section{Structural Similarity Metrics}

Most similarity measures are unable to capture the perceptual similarity of images/video frames under the conditions of varied luminance, contrast or noise. The 
Structural Similarity Measure (SSIM) overcomes these problems [6], which is defined as the distance between two images or sub-images by jointly comparing the mean and variance characteristics. In [7] a complex wavelet domain image similarity measure has been proposed, which is simultaneously insensitive to small luminance change, contrast change and spatial translation.

Due to the symmetric nature of Daubechies complex wavelet, in this paper we are measuring the similarity of the object feature vector using a structural similarity measure in Daubechies complex wavelet domain.

Suppose in the complex wavelet transform domain, $c^{x}=\left\{c_{i, j}^{x} \mid i=1, \ldots, M, j=1, \ldots, N\right\}$ and $c^{y}=\left\{c_{i, j}^{y} \mid i=1, \ldots, M, j=1, \ldots, N\right\}$ are two sets of wavelet coefficients corresponding to two images. Then structural similarity in CxWT domain (CW-SSIM) is given by

$$
S_{c}\left(c^{x}, c^{y}\right)=\frac{2\left|\sum_{i=1}^{M} \sum_{j=1}^{N} c_{i, j}^{x} c_{i, j}^{y^{*}}\right|+k}{\sum_{i=1}^{M} \sum_{j=1}^{N}\left|c_{i, j}^{x}\right|^{2}+\sum_{i=1}^{M} \sum_{j=1}^{N}\left|c_{i, j}^{y}\right|^{2}+k}
$$

Where $\mathrm{c}^{*}$ denotes the complex conjugate of $\mathrm{c}$, and $\mathrm{k}$ is a small positive constant.

\section{The Proposed Algorithm}

In the proposed algorithm the object is localized in a bounding box around the boundary of the object in the reference frame, either manually or by using an automated detector. The feature vector (the coefficients at level $\mathrm{j}$ ) of the image inside the bounding box is calculated in terms of the Daubechies complex wavelet coefficients. The position of the object in the next frame is predicted by motion prediction algorithm as proposed by the authors in [8]. Candidate subframes of the object are generated by shifting the predicted position by $\pm \mathrm{p}$ points. Iteratively comparing the feature vectors using structural similarity, the subframe with maximal similarity is found.

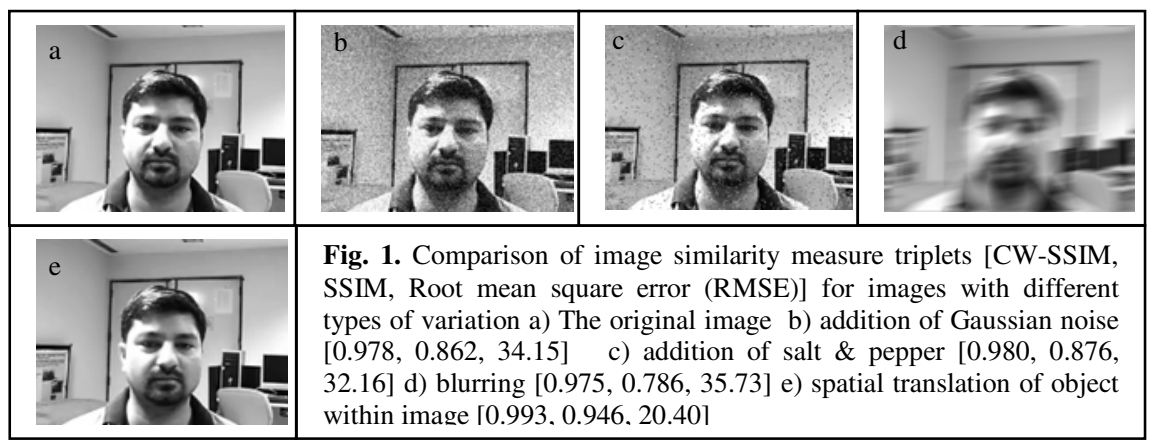

It can be seen from fig. 1 that despite the images having different kind of variations and noise, a high value of similarity using CW-SSIM index (min. 0.975, max. 0.993) is obtained compared to SSIM (min. 0.786, max. 0.946) and RMSE(min 20.40, max. 35.73). This demonstrates the effectiveness of CW-SSIM index as an image similarity measure. 
Table 1. The proposed algorithm

\section{Initialisation}

1. Select object of interest as reference object $\left(\mathrm{O}_{\text {ref }}\right)$

$$
O_{\text {ref }}=\left\{C x W T_{i, j}\right\} i=1 . . . l, j=1 \ldots w
$$

\section{Prediction Step}

2. Predict the position of the object in next frame

\section{Target Localization}

3. Considering the predicted position, define a search space having size $\pm p$ points larger than $O_{\text {ref }}$

4. Compute the feature vector and similarity to get the target position as,

Where

$$
C_{a, b}^{*}=\arg \max \left[S_{a, b}\left(O_{r e f}, C_{a, b}\right)\right]_{a, b=-p}^{a, b=+p}
$$

- $\mathrm{C}_{a, b}$ represents candidates in search space.

- $S_{a, b}$ represents the CW-SSIM in complex wavelet domain as defined in eq. (2).

5. Extract the next frame and Go to step 2 .

\section{Experimental Results and Discussion}

To evaluate the performance of the proposed object tracking method, we have used two videos, where we found significant variations in the object's pose, illumination and background. The first video is recorded in our lab and the second video has been taken from the dataset S1 (camera 3) from IEEE PETS 2006 workshop.

\subsection{Qualitative Evaluation}

For a qualitative comparison of the results of the proposed complex wavelet method (CWT Tracker) with one of the popular algorithms in literature, the Mean Shift method (MS Tracker) [2], we present here some snapshots of the results of both methods on the video sequences (see fig. 2, 3). The results show that CWT Tracker provides good performance as compared to MS Tracker. Fig. 2 shows that the MS Tracker drifts from the actual location of object due to lighting variation in the background (fig 2(b,d)), due to camera motion and change in pose (fig 2(e,f)). MS Tracker has poor performance particularly in the second video, where it leaves the target after a few frames only as shown in fig. 3(c,d), due to same appearance of the background and the object. Our method drifts from the ground truth data as shown in fig. 3(e), only when there is drastic pose and illumination change.

\subsection{Quantitative Evaluation}

To evaluate the tracking accuracy quantitatively, we compare our results with the manually labeled "ground truth" data and compute the Euclidian distance between the centroid positions of the tracked object and the corresponding ground truth data. For 


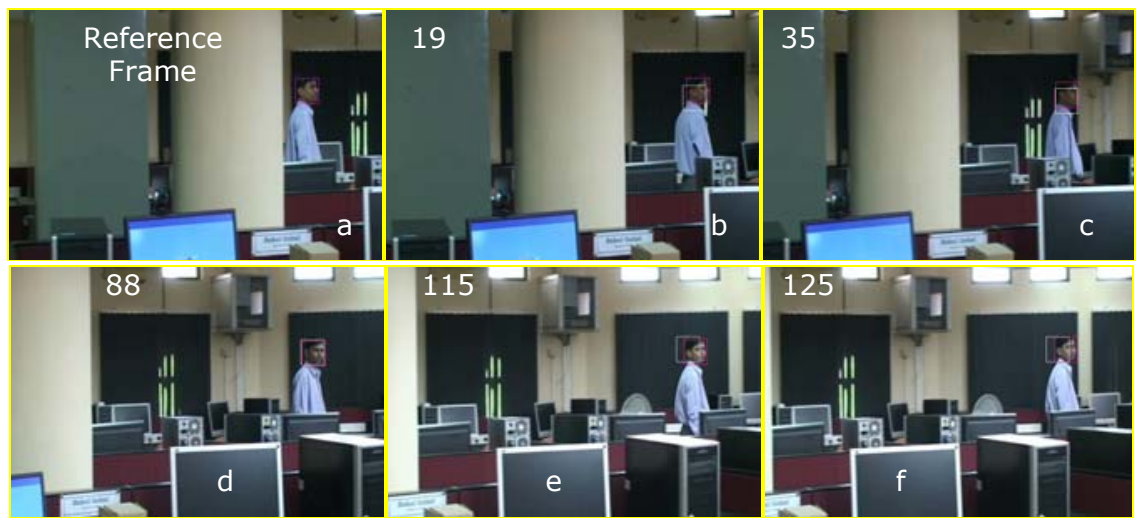

Fig. 2. A comparison of the proposed CWT tracker (indicated with magenta box) with MS tracker (represented by white box) on the video recorded in the lab
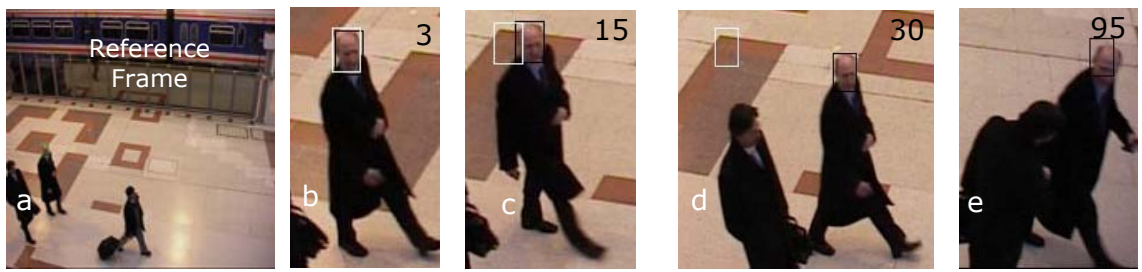

Fig. 3. A comparison of the proposed CWT tracker (indicated with black box) with MS tracker (represented by white box) on PETS 2006 video dataset S1 (camera 3)

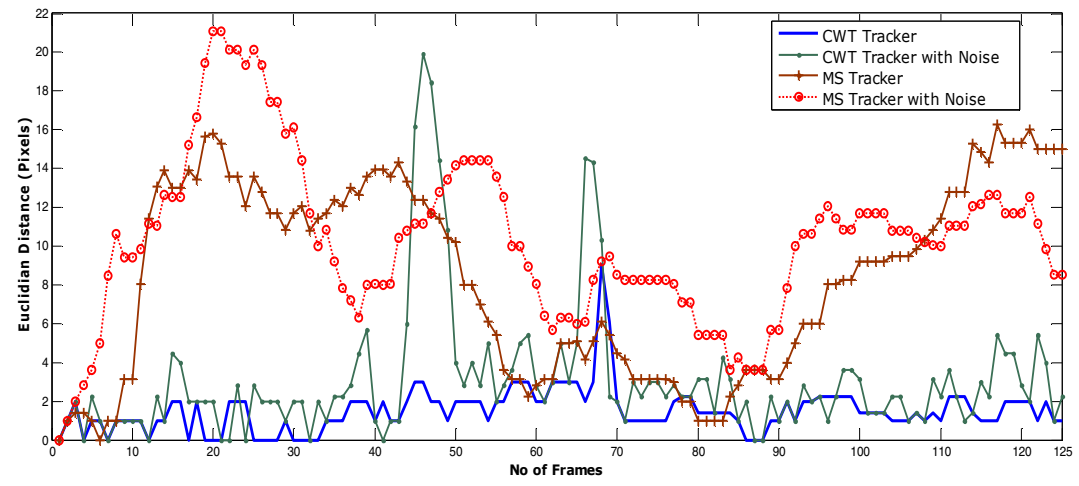

Fig. 4. The error the of object location using the Euclidian distance measure (in number of pixels) for CWT and MS Tracker without and with noise (Gaussian Noise with mean $=0$, variance $=.1$ ) 
each frame of the first video, the error is plotted in fig. 4. For CWT Tracker the tracking position has very small deviation from the ground truth data, the average error in Euclidian distance is 1.52 pixels, while in the case of MS Tracker it is 8.44 pixels. From fig. 4 it is evident that the proposed method gives more accurate results even in the presence of noise (Gaussian Noise with mean=0, variance=.1).

The position, velocity and acceleration information from previous three frames are used to predict the location of the object in the current frame, which gives fairly accurate predictions even for complex motion.

\section{Conclusion}

Due to its approximate shift invariant nature Daubechies complex wavelet transform based method effectively tracks the object in varying real-life environment and even in the presence of noise. In this paper a new robust algorithm based on features and structural similarity is proposed for object tracking. The structural similarity in complex wavelet domain is exploited as similarity measure to make the tracker robust towards small luminance and contrast change and spatial translation. The experiments show that the average localization error for CWT Tracker is 1.5 pixels only. We can extend this work for multiple objects by introducing occlusion handling scheme.

\section{References}

1. Yilmaz, A., Javed, O., Shah, M.: Object Tracking: A Survey. ACM Journal of Computing Surveys 38(4) (2006)

2. Comaniciu, D., Ramesh, V., Meer, P.: Kernel-based Object Tracking. IEEE Transactions on Pattern Analysis and Machine Intelligence 25(5), 564-575 (2003)

3. Khansari, M., Rabiee, H.R., Asadi, M., Ghanbari, M.: Object Tracking in Crowded Video Scenes based on the Undecimated Wavelet Features and Texture Analysis. EURASIP Journal on Advances in Signal Processing, Article ID 243534 (2008)

4. Selsnick, I.W., Baraniuk, R.G., Kingsbury, N.: The Dual-Tree Complex Wavelet Transform. IEEE Signal Processing Magazine, 123-151 (November 2005)

5. Khare, A., Tiwary, U.S.: Symmetric Daubechies Complex Wavelet Transform and its Application to Denoising and Deblurring. WSEAS Transactions on Signal Processing 2(5), 738-745 (2006)

6. Wang, Z., Bovik, A., Sheikh, H., Simoncelli, E.: Image Quality Assessment: from Error Visibility to Structural Similarity. IEEE Trans. on Image Processing 13(4), 600-612 (2004)

7. Wang, Z., Simoncelli, E.P.: Translation Insensitive Image Similarity in Complex Wavelet Domain. In: Proc. IEEE Int. Conf. Acoustics, Speech and Signal Processing, Philadelphia, PA, vol. 2, pp. 573-576 (2005)

8. Jalal, A.S., Tiwary, U.S.: A Robust Object Tracking Method for Noisy Video using Rough Entropy in Wavelet Domain. In: Proceedings of the International Conference Intelligent Human Computer Interaction, pp. 113-121. Springer, India (2009) 